\title{
A NOTE ON DATA PROCESSING IN THE SPLIT HOPKINSON PRESSURE BAR TESTS
}

$\mathbf{T}$ he Split Hopkinson Pressure Bar (SHPB), or Kolsky's apparatus, has been widely used for the study of dynamic behaviors of materials. It was initially developed for use in compressive tests, ${ }^{1}$ and later, was modified for tensile ${ }^{2}$ and torsion ${ }^{3}$ as well as other impact loading configurations. ${ }^{4}$ The concept of the SHPB test involves the determination of dynamic stress, strain and displacement occurring at the end of a bar through observation of the strain at a position some distance away. The experimental method is based on some assumptions, i.e., onedimensional wave in the bars, uniform stress and strain fields in the specimen, and neglecting the effect of specimen inertia. The deviation of real SHPB configuration from the assumptions will result in errors in the measured stressstrain relation. Hence, in spite of its widespread use, it has been the subject of extensive analytical and numerical studies to evaluate the assumptions in order to improve the accuracy of the measured stress-strain curves. reflected wave, $\sigma_{r}$, as well as a transmitted wave, $\sigma_{t}$. In the linear elastic range, the relations between the incident, reflected and transmitted waves are easily obtained from analyzing the conditions of force balance, continuum, and Hooke's law, i.e.,

$$
\sigma_{r}=\frac{1-T}{1+T} \sigma_{i} \quad \sigma_{t}=\frac{2}{1+T} \frac{A_{1}}{A_{2}} \sigma_{i} \quad T=\frac{\rho_{1} c_{1} A_{1}}{\rho_{2} c_{2}}
$$

where $\rho, c, A$ are the density, the wave velocity and the crosssectional area of the bars, respectively. The subscripts represent different bars.

Let us consider a specimen of elastic body in SHPB tests (Fig. 2). The transmitted stress of $\sigma_{T}(t+2 \tau)$ is produced by the stress of $\sigma^{\prime}$, and according to the superposition principle of elastic waves, $\sigma^{\prime}$ is the result of the incident stress of $\sigma_{I}(t$ $+\tau)$ as well as the stress of $\sigma^{\prime \prime}$ which is related to the transmitted stress of $\sigma_{T}(t)$. All the relation between the stresses can be expressed by eq 1 , and the transmitted wave of $\sigma_{T}$ in the output bar is finally expressed as

$$
\sigma_{T}(t+\tau)=\frac{4 F}{(1+F)^{2}} \sigma_{I}(t)
$$

Fig. I: Reflected and transmitted waves at the interface (when $0<t<\tau$ ) move the dispersion effects from measured pulses in order to reconstruct the original load-time history. ${ }^{6}$ After discussing the influence of the wave velocity on the measured stress-strain curves, Lifshitz and Leber proposed a method for accurately determining the wave velocity. ${ }^{7}$ For the dynamic behavior of materials in the range of small strain, Zhao and Gray showed the importance of exact time delays and wave dispersion in data processing of SHPB tests and suggested a SHPB analysis by inverse calculation. ${ }^{8}$

In this paper, an elastic wave propagation analysis in SHPB test is performed so that the governing equation between the incident wave and the transmitted wave is obtained. The relationship is applied to SHPB data processing in order to improve the accuracy of the measured stress-strain relation. The relationship is also applied to analyze the load-time history of the specimen.

\section{ANALYSIS OF THE ELASTIC WAVE PROPAGATION IN SHPB TEST}

In Fig. 1, when a propagating longitudinal stress wave, $\sigma_{i}$, reaches the interface between two bars, it will produce a

$$
\begin{gathered}
\sigma_{T}(t+2 \tau)=\frac{4 F}{(1+F)^{2}} \times \sigma_{I}(t+\tau) \\
+\left(\frac{1-F}{1+F}\right) \sigma_{T}(t) \\
(\text { when } t>\tau) \\
F=\frac{\rho_{0} c_{0} A_{0}}{\rho c A} \quad \tau=\frac{L}{c}
\end{gathered}
$$

where $c$ and $L$ are the length and wave velocity of specimen. The close form of eq 2 is

$$
\begin{aligned}
\sigma_{T}(t) & =\frac{4 F}{(1+F)^{2}} \sum_{n=0}^{\infty} \\
& \times\left\{\left(\frac{1-F}{1+F}\right)^{2 n} \sigma_{I}(t-(2 n+1) \tau) H(t-(2 n\right. \\
& +1) \tau)\}
\end{aligned}
$$

where $H(t)$ denotes the Heaviside unit function. 


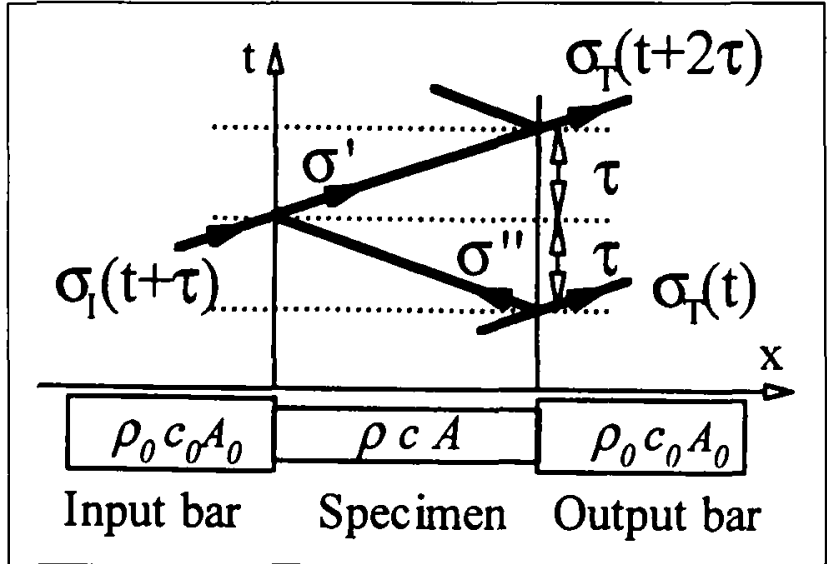

Fig. 2: Wave propagation in SHPB tests for an elastic specimen

Equations 2 and 3 are the relationships between the incident wave and the transmitted wave for an elastic specimen in SHPB tests.

\section{DATA PROCESSING OF SHPB TESTS AND EXPERIMENTAL EXAMINATION}

The SHPB apparatus consists of a striker bar, an input bar and an output bar and associated instruments for recording data is shown in Fig. 3. Equation is the famous formula to calculate the stress and strain of the specimen by using the incident wave, $\varepsilon_{I}(t)$, and the transmitted wave, $\varepsilon_{T}(t)$,

$$
\begin{aligned}
\sigma(t) & =\left(A_{0} / A\right) E_{0} \varepsilon_{T}(t) \quad \varepsilon(t) \\
& =2\left(C_{0} / L\right) \int_{0}^{t}\left[\varepsilon_{I}(\tau)-\varepsilon_{T}(\tau)\right] d \tau
\end{aligned}
$$

where $E_{0}=\rho_{0} c_{0}^{2}$ is Young's modulus of bars.

Before substituting the recorded strain-time relations, $\varepsilon_{I}(t)$ and $\varepsilon_{T}(t)$, into eq 4 , they have to be shifted relatively by the time delays of $T_{1}$ and $T_{2}$ to bar ends or some other positions. When the dynamic behavior in the range of small strain is concerned, such as tests of composites and ceramics, the time delays have a strong influence on the measured stress-

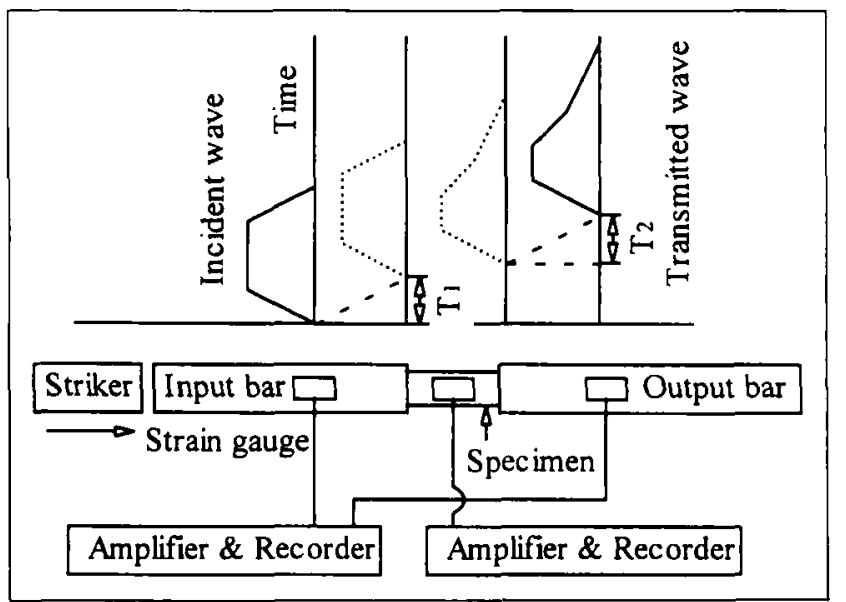

Fig. 3: Schematic of a SHPB test strain curves. Moreover, it is difficult to determine the delay times accurately. ${ }^{7,8} \mathrm{~A}$ curve-fitting method for determining the delay times more accurately was proposed in the reference. ${ }^{8}$ In their analysis, by using the method of characteristic network of elastic wave propagation in the specimen, a simulated transmitted wave is calculated from the incident wave with assuming a fictitious wave velocity of specimen. The simulated transmitted wave is compared with the recorded transmitted wave to determine the time delays. Because the characteristic network depends on the wave velocity as well as the length of specimen, the manipulation is time-consumption work.

However, the curve-fitting method is easy to apply by using eq 2 or 3 as shown in the following. When the specimen density is known, the shape of the transmitted wave only depends on the wave velocity of the specimen according to eq 3 . Hence, presuming a fictitious wave velocity of the specimen, a predicted transmitted wave can be calculated according to eq 2 or 3 as shown in Fig. 4. The shape fitting of the predicted transmitted wave with the measured one in the early stage will result in a rational value of the wave velocity of the specimen. At the same time, the wave shifting of the real transmitted wave will result in a rational value of time delay of $T_{1}+T_{2}$. After obtaining the delay time, the stress-strain curve can be determined according to eq 4 .

SHPB tests of unidirectional composites with different fiber volume fractions and pure matrix have been done in order to check the validity of data processing of SHPB tests based on eq 2. The input and output bars are fabricated from hardened SUS-304 steel with $16 \mathrm{~mm}$ in diameter and $1000 \mathrm{~mm}$ in length, the specimens are unidirectional glass fiber/vinyl ester composites (fiber volume fraction $V_{f}=0.2,0.4,0.6$ ) and pure matrix, and the specimen is of short circular cylindrical shape with $6.6-6.8 \mathrm{~mm}$ in diameter and $4.4-5.5 \mathrm{~mm}$ in length. The specimen strain is independently measured by the strain gauge (gage length is $0.3 \mathrm{~mm}$, KFG-03-120-C111L1M2R, Kyowa, Japan) attached to the specimen (Fig. 3). Figure 5 shows the comparison between the independentlymeasured strain-time curve and the calculated strain-time curve form the above data processing. Figure 6 shows the

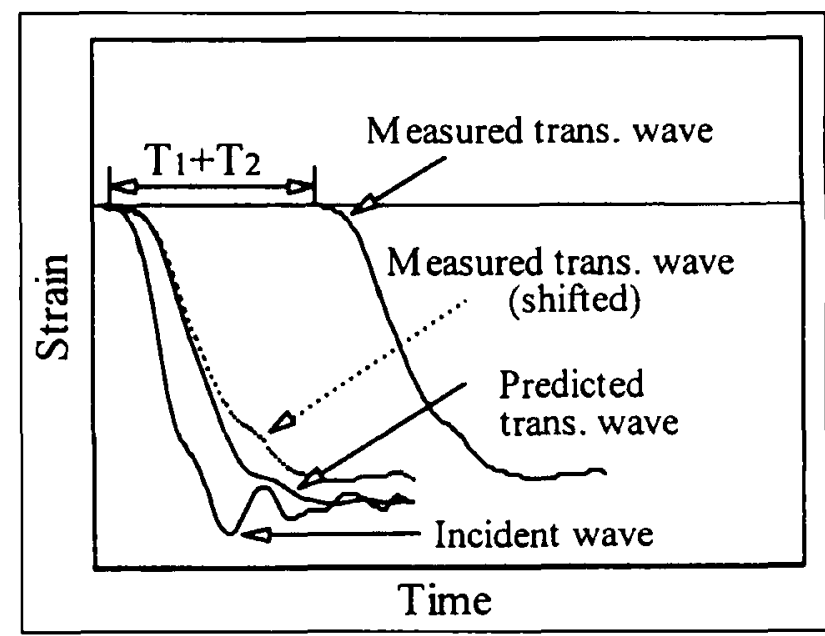

Fig. 4: Method for determining the time delay in SHPB test 


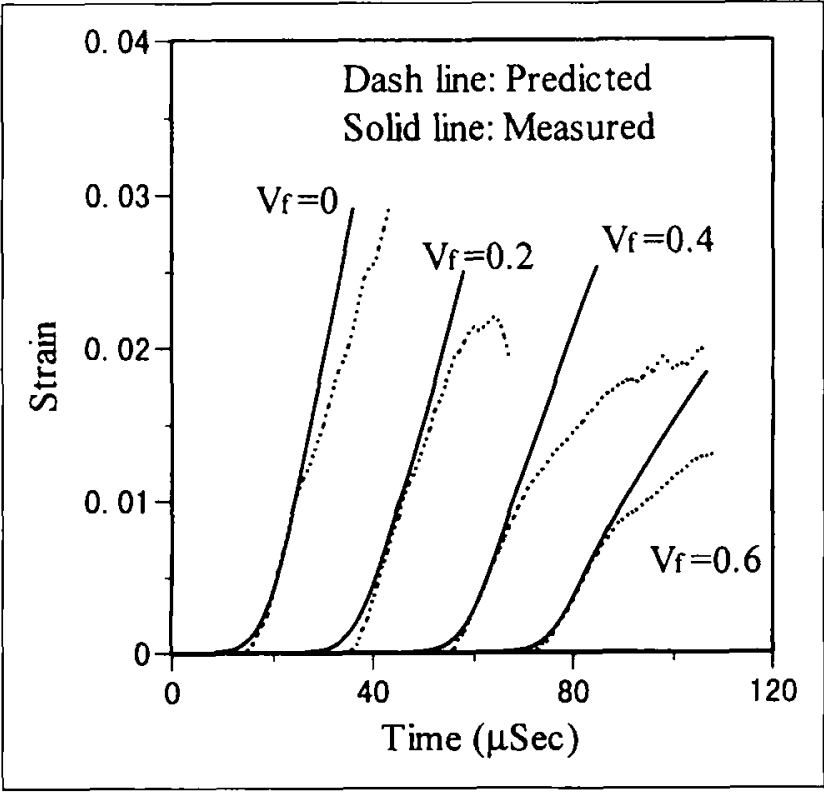

Fig. 5: Comparison between the predicted and the measured strain-time curves

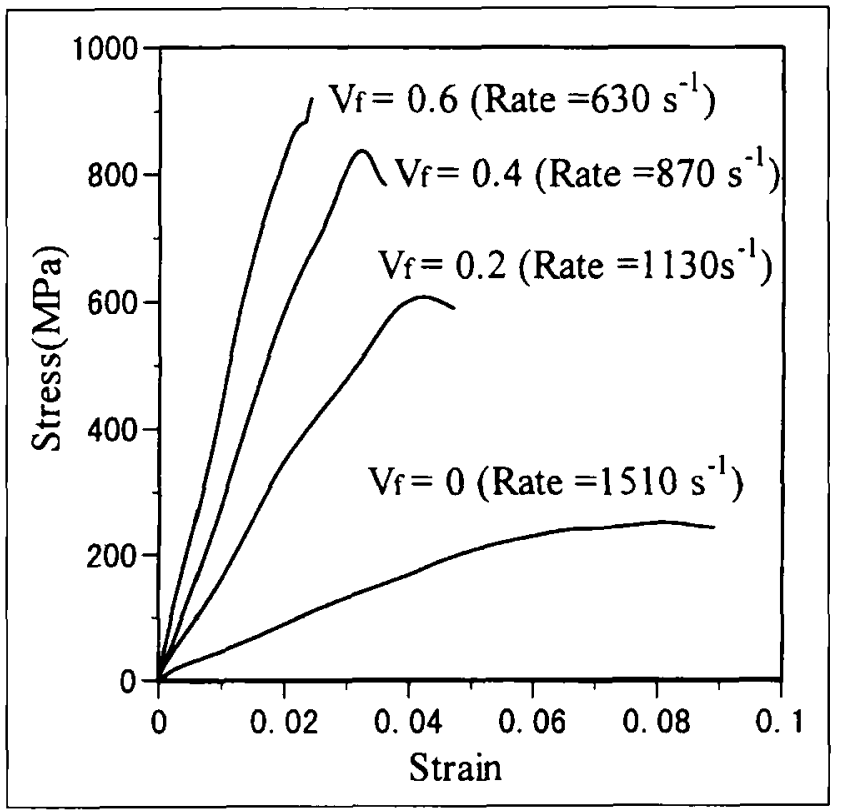

Fig. 6: The measured stress-strain curves of composites and pure matrix

corresponding stress-strain curves. Table 1 shows the parameter values of specimen in experiment and data processing. Here the specimen modulus used in eq $2, E$, is determined from the curve-fitting in Fig. 4 , and $E^{*}$ is the initial modulus in Fig. 6.

From Fig. 5, it can be seen that the strain-time curves are in good agreement within the effective measuring range of the strain gauge (about $1 \%$ in the current tests). It shows that the data processing in the range of small strain is reliable. The initial part of the stress-strain curves in Fig. 6 is
Table I -Parameter Values of Specimens

\begin{tabular}{lcccccc}
\hline$V_{1}$ & $\begin{array}{c}\rho \\
\left(g / \mathrm{cm}^{3}\right)\end{array}$ & $\begin{array}{c}D \\
(\mathrm{~mm})\end{array}$ & $\begin{array}{c}L \\
(\mathrm{~mm})\end{array}$ & $\begin{array}{c}E \\
(\mathrm{GPa})\end{array}$ & $\begin{array}{c}E^{*} \\
(\mathrm{GPa})\end{array}$ & $\mid E^{*}-E / E$ \\
\hline 0.0 & 1.10 & 6.60 & 5.50 & 4.40 & 4.18 & $5.0 \%$ \\
0.2 & 1.33 & 6.75 & 5.00 & 16.3 & 15.6 & $4.3 \%$ \\
0.4 & 1.57 & 6.80 & 4.40 & 29.0 & 29.1 & $0.34 \%$ \\
0.6 & 1.79 & 6.90 & 5.00 & 48.4 & 43.5 & $10.1 \%$ \\
\hline
\end{tabular}

linear, and the discrepancy between $E$ and $E^{*}$ is small. These provide another indirect demonstration.

\section{INFLUENCE OF THE INCIDENT WAVE ON THE SPECIMEN LOAD}

It is empirically and intuitively known that the specimen stress can be varied by changing the amplitude or duration of the incident wave in SHPB tests. In some cases, such as the dynamic recovery SHPB tests where a desired load level of the specimen is expected, ${ }^{4}$ the controlling of the amplitude and duration of incident wave becomes very important. The relationship of eq 3 is helpful to understand how the incident wave influences the load history of specimen.

Supposing a step incident stress wave (Fig. 7), $\sigma_{1}(t)=\sigma_{0}$ $H(t)$ and substituting it into eq 2 , and also replacing $\sigma_{T}(t+$ $2 \tau$ ) with the simplification,

$$
\sigma_{T}(t+2 \tau)=2 \tau \sigma_{T}^{\prime}(t)+\sigma_{T}(t)
$$

because $\tau$ (the duration for elastic wave transmits through a distance of the specimen length) is usually much small, we have

$$
\sigma_{T}^{\prime}(t)=\frac{4 F}{(1+F)^{2}} \frac{\sigma_{0}}{2 \tau}-\frac{4 F}{(1+F)^{2}} \frac{\sigma_{T}(t)}{2 \tau}
$$

with the initial condition, $\sigma_{T}(0)=0$, the solution of eq 5 is,

$$
\sigma_{T}(t)=\sigma_{0}\left\{1-\exp \left[-\frac{4 F}{(1+F)^{2}} \frac{t}{2 \tau}\right]\right\}
$$

It shows that the transmitted wave will increase approximately in an exponential way as the increase of time. Because the specimen stress is $\sigma(t)=A_{0} \sigma_{T}(t) / A$ at the interface

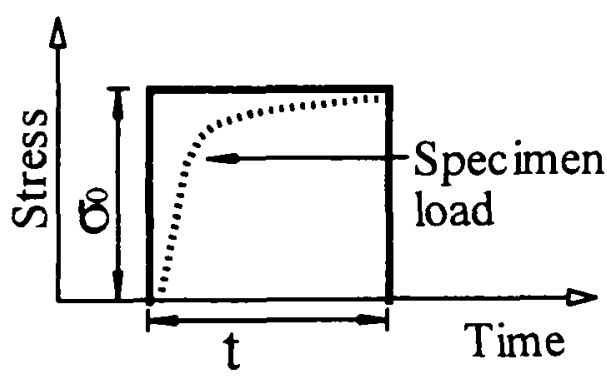

Fig. 7: Incident wave and specimen load 
between bar and specimen, the specimen load-time history is also exponential. Hence, the amplitude of incident wave has a stronger effect on the specimen load than the duration. Moreover, the stress rate, $d \sigma_{T}(t) / d t$, also decreases exponentially from its maximum value at the beginning according to eq 6.

\section{CONCLUSIONS}

In this paper, a close-form relationship between the incident wave and the transmitted wave was deduced on the analysis of elastic wave propagation in SHPB tests for the elastic specimen. The relationship was applied to determine the time delay in data processing of SHPB tests in order to improve the accuracy of the measured stress-strain curve in the range of small strain. This method was examined experimentally to be capable of obtaining the accurate strain-time history in the range of small strain. Moreover, the relationship was also used to analyze how the amplitude and duration of the incident wave influence the specimen loadtime history in the range of elastic response range in SHPB tests.

\section{References}

1. Kolsky, $\mathrm{H}$. An Investigation of the Mechanical Properties of Materials at Very High Rate of Loading, Proc. Phys. Soc. B62 p. 676-700 (1949).
2. J. Harding, Wood, E.D., and Campbell, J.D. Tensile Testing of Materials at Impact Rates of Strain. J. Mech. Engng. Sci. 2, p. 8896 (1960).

3. Duffy, J., Campbell, J.D., and Hawley, R.H. On the Use of a Torsional Split Hopkinson Bar to Study Rate Effect in 1100-0 Aluminum. J. Appl. Mech. 38, p. 83-91 (1971).

4. Nemat-Nasser, S., Issacs, J.B. and Starrett, J.E. Hopkinson Techniques for Dynamic Recovery Experiments, Proc. R. Soc. Lond., A435, p. 371-391 (1991).

5. Davis, R.M. and Hunter S.C. The Dynamic Compression Test of Solids by the Method of the Split Hopkinson Pressure Bar, J. Mech. Phys. Solids. 11, p. 155-179 (1963).

6. Gong, J.C., Malvern, L.E. and Jenkins, D.A. Dispersion Investigation in the Split Hopkinson Pressure Bar, J. Engng. Mater. Tech. 112, p. 309-314 (1990).

7. Lifshitz J.M. and Leber, H. Data Processing in the Split Hopkinson Pressure Bar Tests, Int. J. Impact Engng. 15, p. 723-733. (1994).

8. Zhao H. and Gray, G. On the Use of SHPB Techniques to Determine the Dynamic Behavior of Materials in the Range of Small Strain, Int. J. Solids Structures. 23, p. 3363-3375 (1996).

\section{Residual Stress Analysis Systems \& Service}

\section{We give you choice...}
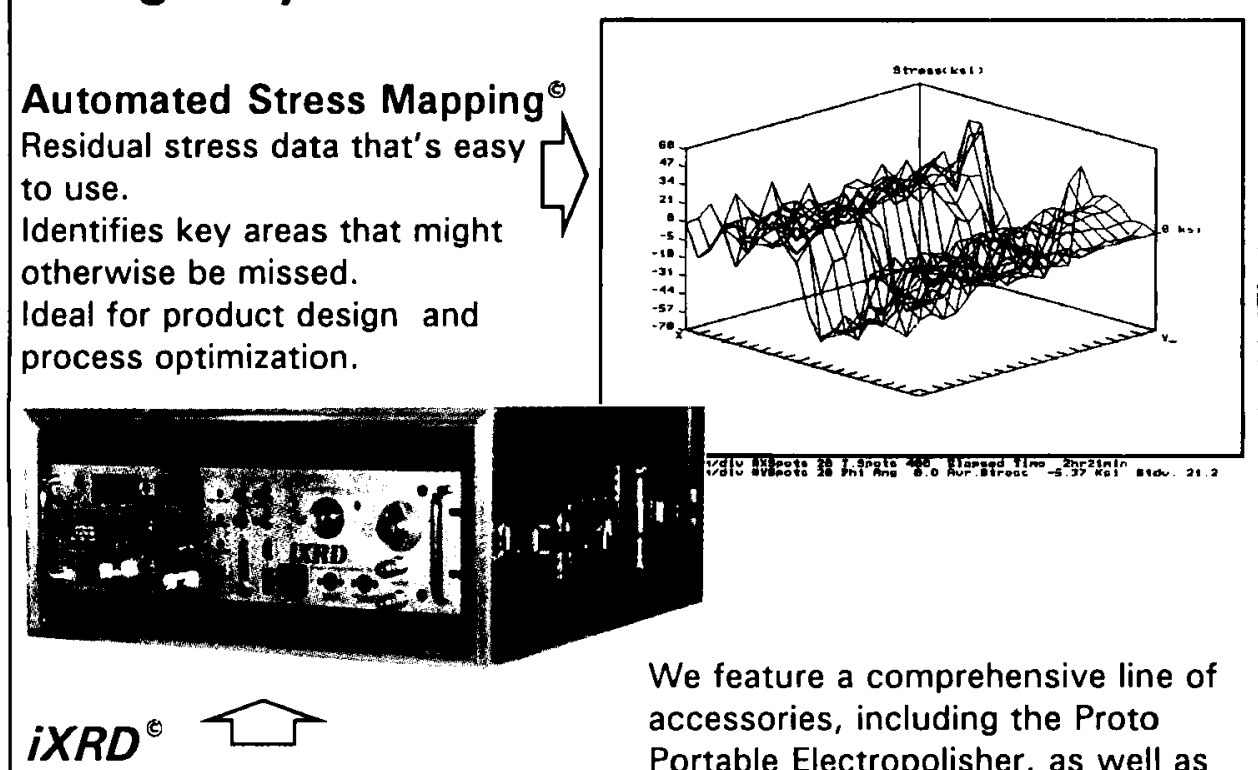

Field Proven, Fast, Accurate, and at 40 Lb. truly Portable

Proto Mfg.Ltd. 2175 Solar Crescent Oldcastle ON Canada
We feature a comprehensive line of accessories, including the Proto Portable Electropolisher, as well as Custom hardware and software.

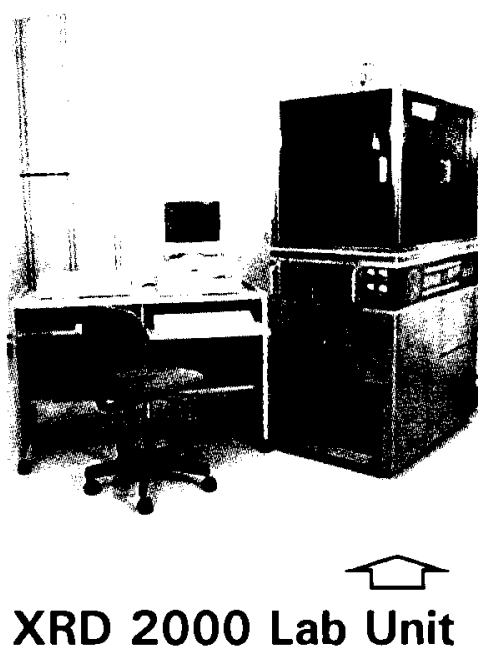

Turnkey solution.

Easy to use, Self-contained. Simultaneous Measurement Stress \& Retained Austenite available as option. 